Carta al Editor.

\title{
Ser representante estudiantil en tiempos de pandemia
}

\author{
Sergio Fernández-Lobo ${ }^{1, *}$, Paloma Arenas ${ }^{1}$ y Jaime Ballesteros ${ }^{1}$ \\ 1 Delegación de estudiantes de Ciencias de la Actividad y del Deporte, Universidad de Alcalá \\ * Autor correspondencia: sergio.fernandezlobo@edu.uah.es
}

DOI: https://doi.org/10.37536/RIECS.2020.5.2.231

Recibido: 23/11/2020; Aceptado: 27/11/2019; Publicado: 30/11/2020

Resumen La comunicación entre Delegados y Profesores, así como los miembros del Equipo Decanal es fundamental, y más en situaciones extrañas, atípicas y sobrevenidas. Es por ello por lo que, con este artículo, trataremos de relatar todos los hechos acontecidos desde la declaración de Estado de Alarma, situación de confinamiento y todo en lo que esto derivó después, hasta final de curso 2019/2020 e inicios del curso 2020/2021. Además de la gestión, tanto académica como personalmente hablando para con los estudiantes del Grado.

Palabras Clave: Pandemia, Representación Estudiantil, Delegación de Estudiantes, COVID-19.

\begin{abstract}
Communication between Delegates and Professors, as well as the members of the Dean's Team is fundamental, and even more so in strange, atypical and unexpected situations. That is why, with this article, we will try to relate all the events that have taken place since the declaration of the State of Alarm, the situation of confinement and everything that derived from it until the end of the 2019/2020 school year and the beginning of the 2020/2021 school year. In addition to the management, both academically and personally speaking to the students of the Degree.
\end{abstract}

Key words: Pandemic, Student Representation, Student Delegation, COVID-19.

La situación sobrevenida del año 2020 hizo tambalear los pilares del mundo en todos los aspectos. Nosotros, como miembros de la Comunidad Universitaria, nos vimos inmersos en diversos procesos a los que no estábamos acostumbrados. A pesar de ello, nos dimos cuenta de que la opinión y trabajo del estudiantado era importante para sobreponernos a tan angustioso proceso. Tuvimos que ponernos al frente, como Delegación de Estudiantes, y dialogar con otros Órganos Universitarios sobre los escenarios en los que nos podíamos mover. ¿El objetivo? Encontrar la mejor manera y más eficaz para finalizar el curso. En numerosas ocasiones se nos dio la oportunidad de comunicarles las decisiones a los estudiantes para llegar a un consenso. Siempre se escuchó nuestra opinión y se nos dieron facilidades a la hora de trabajar.

Cabe resaltar la dureza de todo el proceso en el ámbito académico, en algunas ocasiones vimos como los docentes no podían evitar la pérdida en la enseñanza de competencias de las asignaturas, sobre todo de carácter práctico, por ser un Grado eminentemente práctico. Por otro lado, valorar el esfuerzo de todos y cada uno de los docentes y equipo decanal para poder solventar la situación de la mejor forma posible, todo dentro de los protocolos establecidos por la Universidad.

El concepto de Delegación de Estudiantes según expone la Facultad de Medicina y Ciencias de la Salud de la Universidad de Alcalá en su página Web se puede definir como: "el órgano de representación y coordinación de los estudiantes. Están integradas por los estudiantes que sean miembros de los órganos de gobierno y de representación, así como por los delegados y subdelegados de grupo. Al frente de cada una de ellas, se encontrará un Delegado General elegido por y entre sus 
miembros, que ostentará la representación ordinaria del sector estudiantil de los estudios en el Centro".

Bajo nuestro punto de vista, creemos que se debería añadir a esta definición que, además, los delegados son los encargados de intervenir para paliar los desacuerdos que pueda haber entre profesores y estudiantes, buscar soluciones e intentar asegurar la buena armonía del Grado al que representan durante todo el curso académico. En definitiva, de representación de todos nuestros compañeros.

Si bien, esta labor, no está exenta de problemas no sólo en el concepto en sí de representación sino en los cuantiosos malentendidos que hacia los representantes ocasiona. Más aún en una situación tan límite como la vivida donde el miedo fue un acompañante habitual y la incertidumbre un sentimiento difícil de gestionar unido a la angustia y a la tristeza de lo que estábamos viviendo.

Además, centrándonos en el ámbito personal, fue una etapa dura para todos y cada uno de los miembros de la Comunidad Universitaria. Y para aquellos que, como nosotros, nos sentimos con la obligación de sentir la responsabilidad de nuestra posición de representantes estudiantiles, más aún. Resaltamos en este punto que, el hecho de estar al frente, hizo que muchos de nuestros compañeros acudieran a nosotros buscando soluciones ante situaciones propias que no podíamos resolver. Tenían exigencias hacia con nosotros sin darse cuenta de que éramos un igual. El hecho de no poder ayudar a las personas que te rodean porque no está en tu mano es muy complicado. Hasta incluso frustrante.

Es cierto que, en algunas ocasiones la Delegación de Estudiantes sirve como punto de referencia ante situaciones atípicas, situaciones a las que los estudiantes no están acostumbrados a enfrentarse. En otras, como punto de información para saber sobre cuestiones que les preocupan o necesitan conocer para continuar con su desarrollo adecuado a nivel universitario.

En referencia a este año, la Delegación de Estudiantes del Grado en Ciencias de la Actividad Física y del Deporte de la Universidad de Alcalá ha dado un paso más allá. El año 2020 ha sido difícil en todos los sentidos en cuanto a la vida universitaria se refiere. Desde el principio del mes de marzo fuimos convocados a una reunión con parte del equipo decanal (Decano de la Facultad- Manuel Zapata Rodríguez-, Vicedecana del Grado de Ciencias de la Actividad Física y del Deporte -Beatriz Muros- y la Vicedecana del Grado en Medicina -Lourdes Lledó García-) para informarnos de la situación a la que nos enfrentábamos. Se paralizaban las prácticas externas y se producían modificaciones en actividades docentes de ambos Grados, tanto Medicina como Ciencias de la Actividad Física y del Deporte.

Esa misma tarde, el Ministerio de Educación comunicaba la noticia de la cancelación de actividades académicas presenciales hasta el 26 de ese mismo mes, acción que desencadenaría sensaciones de incertidumbre, tensión y desconocimiento durante los días posteriores. La medida adoptada por la Universidad de Alcalá fue la prolongación del calendario académico durante 15 días. Esta ampliación sería ubicada en el mes de mayo, por lo que muchos profesores tomaron la decisión de mantener su asignatura en "stand by", para recuperar posteriormente esas actividades perdidas. Pocos días después, la Universidad volvió a publicar un comunicado donde se recomendaba que, aunque se fuesen a recuperar las clases a final de curso, durante ese periodo se siguiese con el calendario y las actividades lectivas, convirtiéndolo en online en la medida que se pudiera.

Desde el primer momento la comunicación que manteníamos previamente con los estudiantes, coordinadores de curso y Vicedecanato del Grado, la conservamos de manera satisfactoria, con la salvedad de que se realizaba de manera online y telemática. En este caso, y al ser una situación sobrevenida e inesperada, nos tuvimos que emplear al máximo debiendo encontrar huecos para reuniones virtuales casi diarias en las que recibíamos novedades y sobre todo informábamos de los hechos que estaban ocurriendo entre estudiantes y docentes, exponiendo dichas propuestassensaciones forjadas a partir del resto de los estudiantes del Grado.

Con cada nueva información por parte de las autoridades sanitarias el escenario cambiaba, por lo que estábamos constantemente en un periodo de adaptación. Las decisiones que se tomaban día a día no eran eficaces en la jornada siguiente. Fue una situación caótica, que en el caso de nuestro Grado supimos solventar de una manera bastante coherente para con las necesidades de los estudiantes y docentes. Por un lado, debíamos dar voz a los estudiantes en esta difícil situación, a la vez que 
entender a los docentes que se empleaban al máximo para que adquiriéramos las competencias necesarias de cada asignatura.

La enseñanza y docencia se iba controlando semanalmente mediante reuniones que iban cobrando cada vez más importancia. Los estudiantes, por medio de sus Delegados, coordinadores de curso y Vicedecanato integraban esas reuniones, por lo que se conseguía una óptima coordinación entre todas las partes con conocimiento de lo que iba sucediendo en todas las asignaturas del Grado, y poder así terminar el curso de la mejor manera y con la mejor enseñanza posible, dentro de todo el caos que se generó.

En muchas ocasiones, el hecho de ser el nexo entre estudiantes y docentes suponía un estrés adicional porque nuestra labor era mucho más relevante en aquella situación ya que se nos requería en el papel de estudiantes del Grado y representantes estudiantiles. Además, nosotros nos encontrábamos con problemas personales y/o familiares propios, derivados por la afectación de personas cercanas a nuestro entorno por COVID-19.

Para nosotros era como estar matriculados en una asignatura más, puesto que le dedicábamos el mismo tiempo, o más, a la gestión del Grado, que a la realización de trabajos de otras asignaturas del plan de estudios. De igual manera teníamos la responsabilidad y obligación de transmitir las nuevas informaciones, provenientes de cualquier medio oficial (reuniones, correos, comunicados...), a los estudiantes para que estuvieran al tanto del futuro próximo al que nos enfrentaríamos. En algunos momentos sentimos que nuestro trabajo no era valorado como se debería. $Y$, en momentos sentimos todo lo contrario. En momentos el estrés llegó a ser indescriptible, en momentos la calidez de las relaciones humanas marcó situaciones inolvidables. Ambas caras de la moneda igual de importantes para trazarnos un aprendizaje que seguro será vital en nuestro futuro. Ambas caras de la moneda nos hicieron madurar tanto en el plano personal como en la condición de representantes estudiantiles.

Nadie en este 2020 pensaría que nuestro día a día cambiaria de la noche a la mañana, viéndonos inmersos en una crisis sanitaria descolocando así aquellas acciones cotidianas que pasaban desapercibidas y no fuimos conscientes hasta que tuvimos que prescindir de ellas. Esta crisis afectó de manera casi inmediata a la economía, a la sociedad, a la sanidad, y también a la educación. Una educación que estaba acostumbrada a la presencialidad, al contacto directo en las relaciones de profesores y alumnos, a escuchar al docente desde la pizarra y no a través de una pantalla, a compartir espacio de estudio con el resto de los compañeros y no a través de plataformas digitales o videollamadas grupales. Es por ello, que tanto la responsabilidad y trabajo diario de la representación estudiantil, de personas, como los que firmamos este escrito, con nombre y apellidos, junto con los demás órganos educativos, han sido claves en esta "nueva normalidad".

(C) 2020 por los autores; Esta obra está sujeta a la licencia de Reconocimiento 4.0 Internacional de Creative Commons. Para ver una copia de esta licencia, visite http://creativecommons.org/licenses/by-nc-nd/4.0/. 